\title{
Orbits of Open Star Clusters NGC 6067, NGC 2506 ANG IC 465
}

\author{
Geeta Rangwal $^{1}$, R. K. S. Yadav ${ }^{2}$ and Alok K. Durgapal ${ }^{1}$ \\ ${ }^{1}$ Department of Physics (UGC-Centre of Advanced Study), Kumaun University, Nainital, India. \\ ${ }^{2}$ Aryabhatta Research Institute of Observational Sciences, Manora Peak, Nainital, 263001, India \\ Email: geetarangwa191@gmail.com
}

\begin{abstract}
The studies of the orbits of star clusters are very important to understand the evolution of these objects. The survival or dissolution of star clusters in galactic tides within which they orbit depends on their orbit history. The orbits of three galactic clusters namely NGC 6067, NGC 2506 and IC 4651 are calculated using newly available proper motion data taken from GAIA data. These orbits are traced backwards in time which is equal to the age of the clusters. The main parameters of the orbits such as eccentricity, apogalactic distance and perigalactic distance are calculated.
\end{abstract}

Keywords: Star Clusters, Orbits, Photometry and star cluster parameters.

\section{Introduction}

Open star clusters (hereafter Ocs) are the group of loosely bound stars, which have formed by the same molecular cloud. They are concentrated towards the Galactic disc. Hence provide valuable information concerning star formation processes and are key objects for the study of galactic structure and evolution. They move in a circular path around the Galactic center in a same direction. Open star clusters orbit near the Galactic plane, so constantly disturbed by the tidal forces originating from Galactic disc. Due to this, the structure of Ocs are changed. Study of orbits of clusters predicts the survival or dissolution of star clusters in these Galactic tides. We can study the motion of objects at different parts of Galaxy using their orbits.

In present analysis we derived the orbits of three open star clusters NGC 6067, NGC 2506 and IC 4651 using the Galactic mass models. We have also calculated values of eccentricity, apogalactic distance and perigalactic distance for these orbits. In section 2, we have discussed the data used for this study. In section 3, we described the method of orbit calculation. In section 4 , the results and conclusions are discussed.

\section{Data}

The photometric data for the clusters NGC 6067, NGC 2506 and IC 4651 is taken from WFI@2.2 catalogue. The images from WFI@2.2 were reduced using img2xym_WFI software

(Anderson et al. 2006). The kinematical data such as proper motion, radial velocity and parallax was taken from GAIA DR2 catalogue for all three clusters under study.

\section{Calculation of orbits}

Galaxy is devided in three regions; central bulge, Disc and Halo. The distribution of mass in these regions is different from each other. Galaxy models are created on the basis of rotation curve and the perpendicular force. Structure of the Galaxy is not well known yet, so mass models keeps on changing. The total Gravitational potential is the sum of the gravitational potential of bulge, disc and halo. 
To simplify the calculations, we used axissymmetrical Gravitational potential models. For the disc region we used Miyamoto-Nagai (1975) potential. For Galactic bulge Hernqist (1990) potential is used. Binney and Termaine (2008) potential is used for the halo region of the Galaxy.

The central coordinates of the clusters are calculated using the photometric data of clusters. We have calculated the average proper motion, parallax angle and radial velocity for the clusters using star count method. Heliocentric distances for the clusters are calculated using the parallax angles. For orbit determination, we have converted the cluster parameters from equitorial coordinate system to Galactocentric coordinate system. After this the components of gravitational forces are calculated by differentiating the potentials.

Orbits are calculated by integrating the equation of motion backward in time, which is equal to the age of the cluster. The orbits of the clusters under study are shown in Fig.1. This figure shows two dimensional view of the path traced by open clusters, around the Galactic center.

\section{Results and conclusion}

We traced the path of the cluster around the Galactic center. It is evident from the Fig.1 that NGC 6067 being very young cluster has not completed even one revolution around the Galactic center. Orbits of all the clusters in present analysis are found to be circular in shape. We can locate the birth place and present day position of the clusters in Galaxy, using these diagrams. The eccentricity for the clusters NGC 6067, NGC 2506 and IC 4651 is calculated as $0.08, \quad 0.016$ and -0.004 respectively. Apogalatic distances are calculated as $6.164 \mathrm{kpc}, 13.237 \mathrm{kpc}$ and 7.524 $\mathrm{kpc}$ respectively. Perigalatic distances are calculated as $5.237 \mathrm{kpc}, 12.806 \mathrm{kpc}$ and 7.598 kpc respectively.
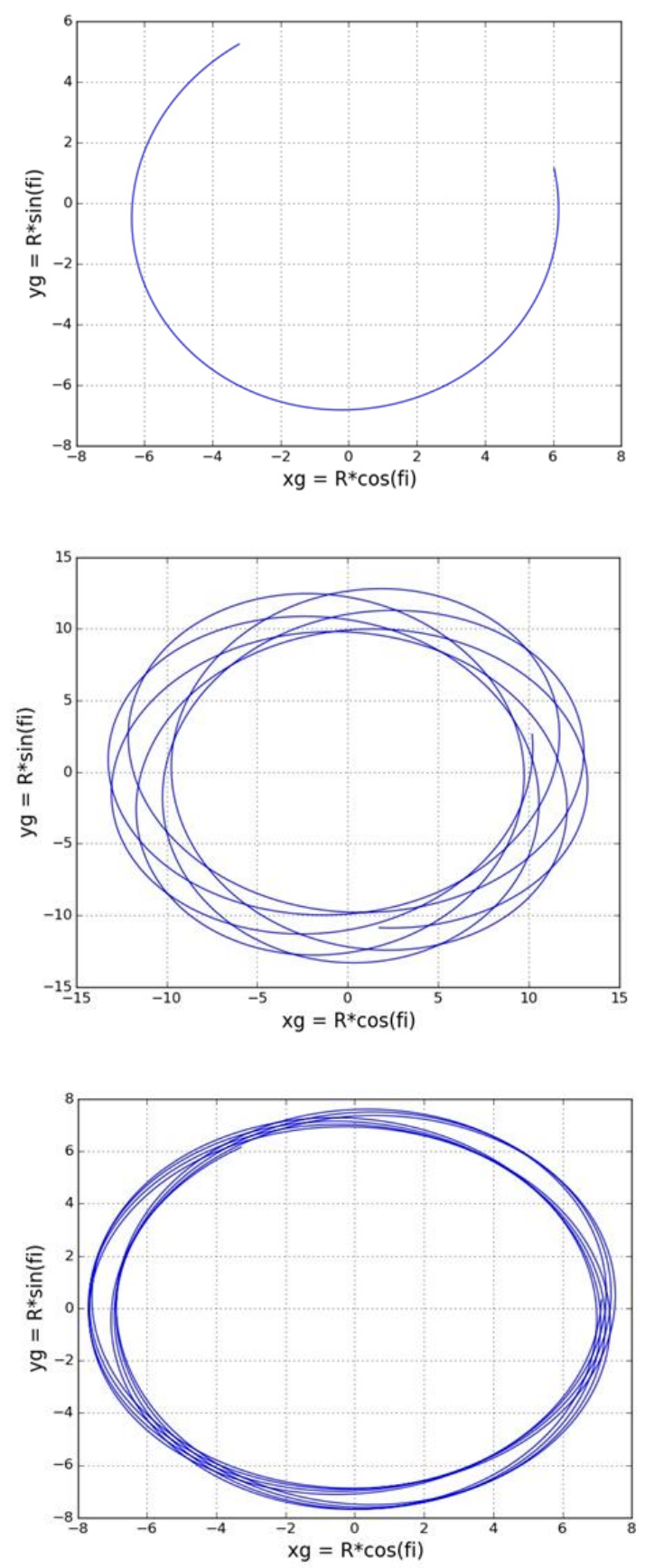

Fig.1: The two dimensional view of the orbits of Galactic star clusters NGC 6067 (upper left), NGC 2506 (upper right) and IC 4651 (lower right). 


\section{Acknowledgements}

This analysis has made use of WFI@2.2 and GAIA DR2 data.

\section{References}

Anderson, J., Bedin, L. R., Piotto, G., Yadav, R. K. S., Bellini, A., 2006, A\&A, 454, 1029

Binney, J., Termaine, S., 2008, Princeton University press.

Hernqist, L., 1990, ApJ, 356, 359.

Miyamoto, M., Nagai, R., 1975, PASJ, 27, 533. 